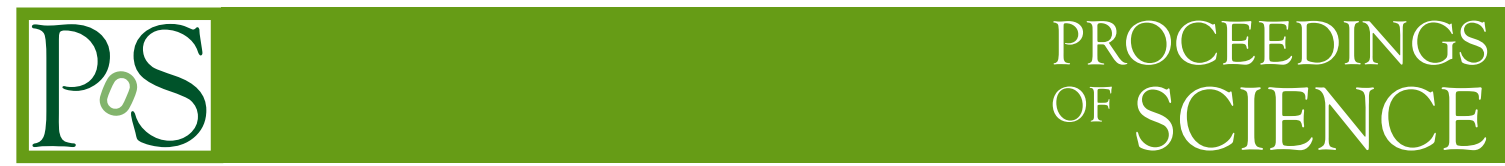

\title{
Standard Model results (excl. top) from CMS at 13
} TeV

\section{Jesús Vizán on behalf of the CMS Collaboration*}

Universidad de Oviedo (ES)

E-mail: Jesus.Manuel.Vizan.Garcia@cern.ch

\begin{abstract}
An overview of the first results at the CMS experiment using proton-proton data at a center-ofmass energy of $13 \mathrm{TeV}$ during the Run II of the LHC is presented. These results comprise aspects such as soft QCD physics, with the measurement of the pseudorapidity distribution of charged hadrons, or the first observation of the main candles for electroweak and flavor physics. The first results regarding top-quark physics obtained by the CMS collaboration, such as the measurements of the $t \bar{t}$ production cross section, are not discussed in this contribution.
\end{abstract}

8th International Workshop on Top Quark Physics

14-18 September, 2015

Ischia, Italy

\footnotetext{
${ }^{*}$ Speaker.
} 


\section{Introduction}

Since the recent start of the Run II of the Large Hadron Collider (LHC), with the first circulating beams seen on the $5^{\text {th }}$ of April of 2015, and the first stable proton-proton beams at a new record collision energy of $\sqrt{s}=13 \mathrm{TeV}$ on the $3^{\text {rd }}$ of June, the Compact Muon Solenoid (CMS) experiment recorded during the Summer approximately $42 \mathrm{pb}^{-1}$ of data passing qualification requirements with the magnetic field on. This small amount of data has served first to commission successfully the CMS detector, which overall shows an excellent performance for this early stage of the 2015 running period $[1,2,3]$. This represents a necessary step to pursue the ambitious CMS physics program during the Run II, being some of its aspects, like measurements in the QCD and electroweak sectors, or flavor physics discussed here.

The first CMS physics measurement submitted for publication, the measurement of the charged hadron pseudorapidity distribution [4] and the first observation of key candles for electroweak and flavor physics are presented in the next sections.

\section{Charged hadron pseudorapidity distribution}

QCD interactions present a unique interest within the standard model, exhibiting remarkable differences with respect to the electromagnetic force, with gluons that interact among themselves, and the confinement of strongly interacting particles at low energy. The understanding and modeling of QCD interactions, in addition of being interesting by itself, impacts directly the potential for precision measurements and searches for new physics at LHC.

Soft particle production cannot be calculated reliably using perturbative QCD and is generally described by phenomenological models, which are implemented in the Monte Carlo (MC) simulations of particle collisions and describe phenomena such as underlying events, or minimum bias and multiple parton interactions. An exhaustive set of measurements performed at hadron colliders are necessary to adequately tune those models. One of those important measurements is the one of the charged hadron pseudorapidity distribution, a basic observable in high-energy particle collisions which gives a handle on the relative weight between soft interactions and contributions from hard-scattering processes, being a relevant measurement for a precise modeling of pile-up collisions.

This measurement was performed using the data recorded by CMS at a center-of-mass-energy of $13 \mathrm{TeV}$ in May 2015, with LHC running with a special low-intensity beam configuration that resulted in a very small proton-proton interaction probability per bunch crossing of $\sim 0.2-5 \%$. Approximately 11.5 million events were acquired by CMS operated at zero magnetic field due to a cryogenic problem, by means of zero bias trigger which required a coincidence of signals from both beam pickup for timing devices. Two complementary methods were employed, based on different reconstruction techniques of the charged-particle trajectories using the hits detected by the CMS pixel detector. The tracklet method, which uses hit pairs, performs a background subtraction using control samples in data; while the track method, which uses hit triplets, minimizes background contributions by requiring an additional hit in the detector. The presence of at least one reconstructed interaction vertex is required in both cases. Both analyses are combined to produce a single measurement, improving the robustness with respect to the individual ones, as different 
aspects related to systematic uncertainties, such as detector misalignment and response, or the dependence with the MC event generators affect the techniques to different extents.

After corrections from detector-level event selection to hadron-level event definitions using MC simulations with PYTHIA 8 v208 (tune CUETP8M1) and EPOS LHC (tune LHC) the multiplicity of primary charged hadrons as a function of the pseudorapidity, $\mathrm{d} N_{c h} / \mathrm{d} \eta$, measured by the tracklet and track methods agreed within $2 \%$ for the central region and within $3 \%$ for forward pseudorapidities, what supports the choice of combining both results.

The results for the combined measurement are shown in Figure 1 (left). In the region $|\eta|<0.5$, the average pseudorapidity density, $\mathrm{d} N_{c h} /\left.\mathrm{d} \eta\right|_{|\eta|<0.5}$, is found to be $5.49 \pm 0.01$ (stat) \pm 0.17 (syst). While the central measured value agrees well with the predictions from both PYTHIA8, with the CUETP8S1 and CUETP8M1 tunes, and from EPOS LHC, the measured distributions in the full $\eta$ range is described more accurately with EPOS LHC. The dependence of $\mathrm{d} N_{c h} /\left.\mathrm{d} \eta\right|_{|\eta|<0.5}$ with the center-of-mass energy is shown in Figure 1 (right). The CMS result at $\sqrt{s}=13 \mathrm{TeV}$ is presented together with previous measurements at lower energies from other experiments. The experimental values are fitted using a second-order polynomial in $\ln (s)$, obtaining a good description over the considered range. The results are compared with the predictions from PYTHIA8 CUETP8S1 and EPOS LHC, which globally reproduce the collision-energy dependence of hadron production in inelastic proton-proton collisions.
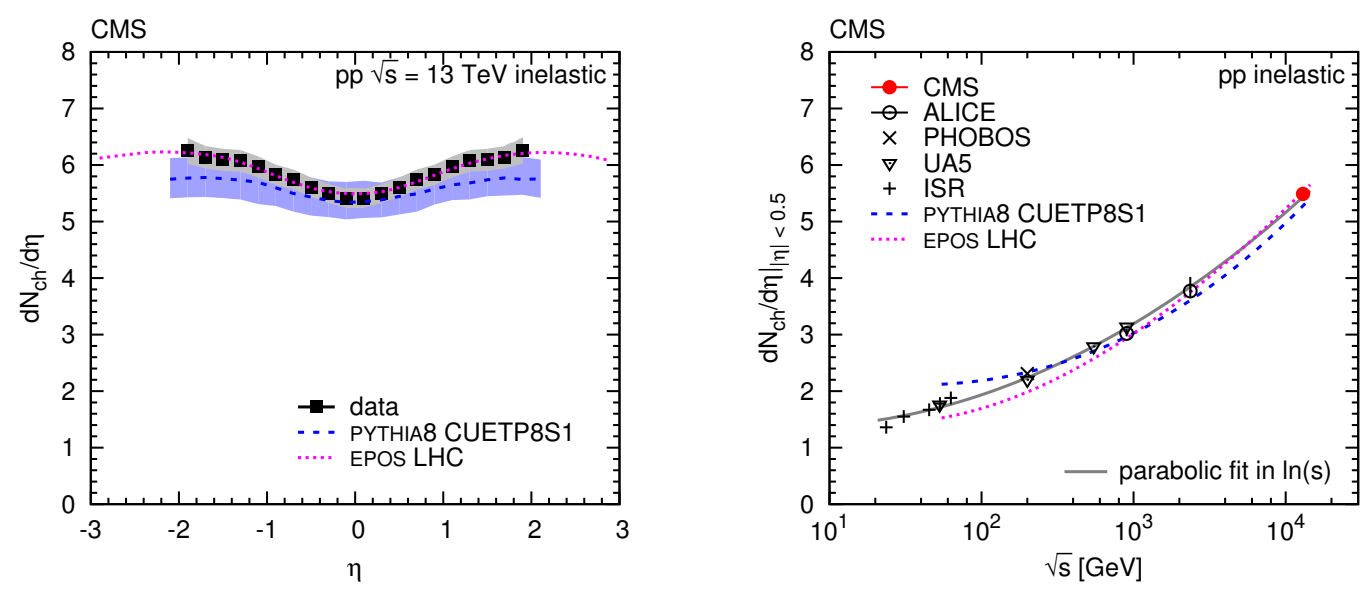

Figure 1: Left: Distribution of $\mathrm{d} N_{c h} / \mathrm{d} \eta$ for inelastic collision events measured in data combining the track and tracklet results (solid markers). The measurement (symmetrized in $\eta$ ) is compared with the predictions by PYTHIA8 CUETP8S1 and EPOS LHC (curves). The grey shaded area encompassing the data points indicates their correlated systematic uncertainties. The blue band corresponds to the envelope of the uncertainties of the CUETP8S1 tune. Right: Dependence of $\mathrm{d} N_{c h} /\left.\mathrm{d} \eta\right|_{|\eta|<0.5}$ with the center-of-mass energy. The solid curve shows a second-order polynomial in $\ln (s)$ fit to the data points. The dashed and dotted curves show the PYTHIA8 CUETP8S1 and EPOS LHC predictions, respectively.

\section{Flavor physics}

The study of flavor physics in hadron collider presents multiple motivations, including its potential to test of QCD and effective theories, its importance to obtain an accurate description 
of the environment where direct searches for new physics are performed, and its provision for a multitude of measurable processes for which precise standard model predictions are available, which become excellent probes to look for indirect evidence for physics beyond the standard model (BSM), even above the direct reach of the LHC.

Despite being a general-purpose detector, CMS presents excellent capabilites for heavy-flavor physics, due to its excellent vertex reconstruction, momentum resolution of its tracker and muon system, and its very flexible trigger system. Already with the data collected during Summer of this year, in the early running stages of Run II of the LHC, resonances corresponding to different quarkonia and heavy-flavor mesons have been seen using dedicated dimuon trigger paths with invariant masses centered around them. Some examples are the $J / \psi$ and $\Upsilon(\mathrm{nS})$ mesons, which are clearly reconstructed using soft muon identification offline requirements. More complex meson decays are also clearly seen applying additional offline requirements. For instance, $B^{ \pm} \rightarrow \mathrm{J} / \psi K^{ \pm}$ and $B^{0} \rightarrow \mathrm{J} / \psi K^{* 0}$ decays are shown in Figure 2 triggering on a displaced $\mathrm{J} / \psi$ plus a track. All these results and additional ones described in [5] give an idea of the impressive potential of CMS for flavor physics during the Run II.
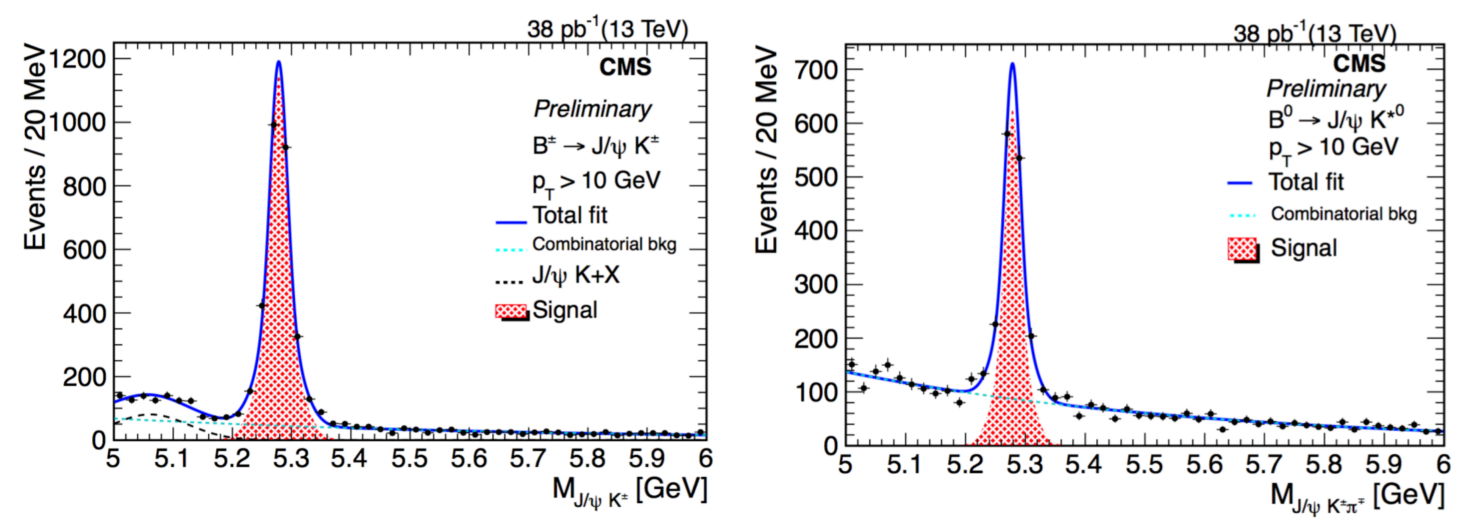

Figure 2: $\mathrm{J} / \psi K^{ \pm}$(left) and $\mathrm{J} / \psi K^{ \pm} \pi^{\mp}$ (right) invariant mass distributions applied to events passing an online selection based on the presence of a displaced $\mathrm{J} / \psi$ and a track after applying additional offline selection criteria.

\section{Electroweak physics}

The Run I left an extraordinary legacy in the electroweak sector. Just a few examples are the measurement of the cross section of vector bosons produced in association with jets, or measurements in multiboson final states. Extensive studies were performed for single $W / Z$ production, a dominant background for many new physics searches and a very sensitive process to several aspects of QCD phenomenology. An overall good agreement with the SM was found for many different final states, like $W+$ up to $\geq 6 \mathrm{j}, W+\mathrm{c}, W+2 \mathrm{~b}, Z+\geq 4 \mathrm{j}, Z+\geq 1 \mathrm{~b}$, or $Z+\geq 2 \mathrm{~b}$ spanning over many orders of magnitude. Multiboson production represents as well another important test of the SM, being as well particularly sensitive to new physics, with the potential of finding hints of it via the study of anomalies in the vector boson couplings. During the Run I, diboson production was 
studied for all the possible combinations of $W, Z$, and $\gamma$. No remarkable deviations from the SM prediction were found and stringent limits on anomalous couplings were set.

The $13 \mathrm{TeV}$ Run will allow to go beyond the precision achieved during the Run 1 and open the possibility of studying in detail new important processes, such as electroweak scattering, key to probe the nature of the Higgs boson. The ground for these goals is starting to be set, for instance with the observation of the basic electroweak candle, the $\mathrm{Z}$ boson, as illustrated in Figure 3, both for $Z \rightarrow e e$ and $Z \rightarrow \mu \mu$.
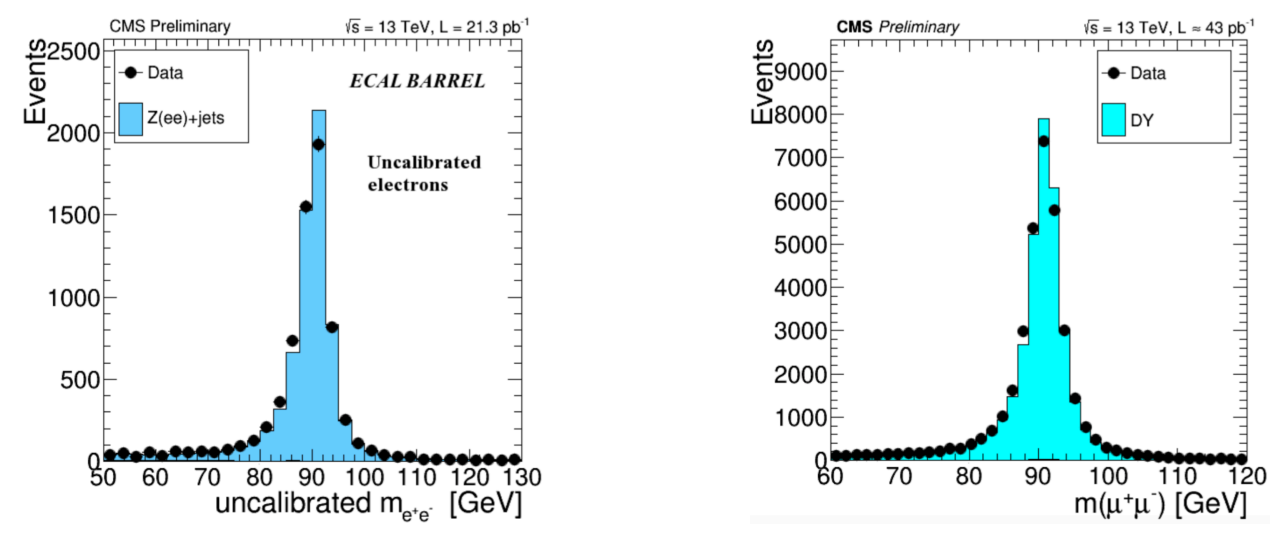

Figure 3: Dielectron (left) and dimuon (right) invariant mass distribution obtained with early CMS Run II data at $13 \mathrm{TeV}$.

\section{Conclusions}

After a very successful Run I of the LHC, marked by the discovery of a Higgs boson with a mass of approximately $125 \mathrm{GeV}$, the standard model will be tested with an unprecedented level of precision in new unexplored territories during the Run II, setting as well the ground for new physics searches. The first months of data taking of the Run II have served not only to commission all the objects used in the different analyses, but also to rediscover the basic SM candles and resonances and produce the first physics measurements. Those achievements represents already an important first step in the development of the CMS physics program for the Run II.

\section{References}

[1] CMS Collaboration, Electron and Photon performance with 50ns Run II data at sqrt(s)=13 TeV, CMS-DP-2015-013 .

[2] CMS Collaboration, Muon Reconstruction and Identification Improvements for Run-2 and First Results with 2015 Run Data, CMS-DP-2015-015.

[3] CMS Collaboration, Additional Performance Results for EPS 2015, CMS-DP-2015-016.

[4] CMS Collaboration, Pseudorapidity distribution of charged hadrons in proton proton collisions at $\sqrt{s}=13 T e V$, Phys. Lett. B 751 (2015) 143 [arXiv:1507.05915].

[5] CMS Collaboration, Selected Heavy Flavor distributions from CMS with first $13 \mathrm{TeV}$ data, CERN-CMS-DP-2015-018. 